\title{
Estimation of the Remaining Useful Life by using Wavelet Packet Decomposition and HMMs
}

\author{
D. A. Tobon-Mejia ${ }^{a, b}$, K. Medjaher, N. Zerhouni \\ ${ }^{a}$ FEMTO-ST Institute \\ AS2M department, UMR CNRS 6174 - UFC / ENSMM / UTBM \\ 25000 Besançon, France \\ (+33) 381402797 \\ diego.tobon@femto-st.fr, kamal.medjaher@ens2m.fr \\ noureddine.zerhouni@ens2m.fr
}

\author{
G. Tripot \\ ${ }^{b}$ ALSTOM Transport \\ 7, avenue De Lattre De Tassigny \\ BP 49, 25290 Ornans, France \\ (+33) 381624437 \\ gerard.tripot@transport.alstom.com
}

\begin{abstract}
This paper deals with an estimation of the Remaining Useful Life of bearings based on the utilization of the Wavelet Packet Decomposition (WPD) and the Mixture of Gaussians Hidden Markov Models (MoG-HMM). The raw data provided by the sensors are first processed to extract features by using the wavelet packet decomposition. This latter provides a more flexible way of time-frequency representation and filtering of a signal, by allowing the use of variable sized windows and different detail levels. The extracted features are then fed as inputs of dedicated learning algorithms in order to estimate the parameters of a mixture of Gaussian Hidden Markov Model. Once this learning phase is achieved, the generated model is exploited during a second phase to continuously assess the current health state of the physical component and to estimate its remaining useful life with the associated confidence value. The proposed method is tested on a benchmark data taken from the "NASA prognostic data repository" related to several bearings'. Bearings are chosen because they are the most used and also the most faulty mechanical element in some industrial systems and process. Furthermore, the method is compared to a traditional timefeature prognostic and some simulation results are given at the end of the paper.
\end{abstract}

\section{TABle of Contents}

1 InTRODUCTION $\ldots \ldots \ldots \ldots \ldots \ldots \ldots \ldots \ldots \ldots \ldots, 1$

2 BACKGROUND............................. 3

3 WPD/MOG-HMM BASED PROGNOSTIC METHOD 4 4 APPLiCATION AND SIMUlation RESUlts ...... 6

5 Conclusions $\ldots \ldots \ldots \ldots \ldots \ldots \ldots \ldots \ldots \ldots \ldots . \ldots \ldots$

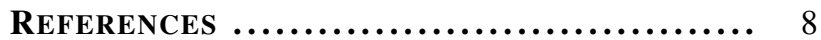

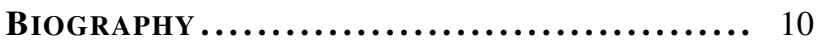

\section{INTRODUCTION}

The competitiveness requirement in industry involves the improvement of the availability, the reliability, the security while reducing the life cycle cost of the production means. One of the possible solutions that can contribute to fulfill

\footnotetext{
1 978-1-4244-7351-9/11/\$26.00 @2011 IEEE.

2 IEEEAC Paper \#1141, Final Version, Updated December 8, 2010.
}

these objectives consists in acting on the maintenance by implementing new maintenance strategies like the Condition Based Maintenance (CBM) [1,2] and reducing the traditional corrective and preventive maintenances.

The CBM is based on the exploitation of the data provided by the monitoring system which continuously tracks the health state of the system. The data gathered are processed and transformed to relevant knowledge in order to estimate the current state of the system and to predict its future one, and thus helping the maintainers to take adequate decisions at appropriate time.

The input data in the prognostic process are signals, where the degradation information is usually masked by noise. To solve this problem and provide the correct information to perform failure prognostic, several methods have been proposed, based in the time domain behavior of the signal, or on traditional spectral analysis. Time domain uses statistical properties from the raw signal as RMS, Kurtosis or crest factor to track failures with some limited success in localized defects [3]. Frequency analysis are widely used in vibration signals for bearing fault detection [4]. The Fast Fourier Transform (FFT) is the simplest frequency domain analysis method. This method is the examination of high-frequency resonances caused by a fault in the spectrum of the signal. However the impact vibration generated by a bearing fault has relatively low energy, it is often overwhelmed by noise with higher energy and noise from the environment. In order to overcome FFT problems, recent advanced signal processing methods such as Short-Time-Fourier-Transform, WignerVille and wavelet analysis have been used. Wavelets have been established as the most widespread tool in many areas of signal processing, due to their flexibility and their efficient computational implementation [5].

Failure prognostic activity aims at anticipating the failure date by predicting the future health state of a given system and its Remaining Useful Life (RUL). According to the International Standard Organization [6], failure prognostic corresponds to the "estimation of the time to failure and the risk for one or more existing and future failure modes". Several other definitions have been proposed in the literature [1,7-10] during this last decade. All the reported definitions agree on prediction step and the estimation of the time before the failure. This time is called RUL in some of them, Estimated Time To Fail- 
ure (ETTF) in the ISO's one [11] and in a small number of publications $[12,13]$ it is defined as a probability that a machine operates without a fault up to some future time.

Contrary to fault diagnostic which is a mature activity, well developed and spread within the research and the industrial communities, failure prognostic is a new research domain $[1,14,15]$. However, the increasing interest accorded to failure prognostic has led to numerous methods, tools and applications during the last decade. According to what is reported in the literature $[1,10,16]$ and taking in account the latest contributions in the field, failure prognostic methods can be classified into three main approaches: model-based, experiencebased and data-driven prognostic, see Fig.1. In addition to these three categories, other sub-categories resulting from the intersection between the main ones has been developed.

The methods of the model-based prognostic approach are

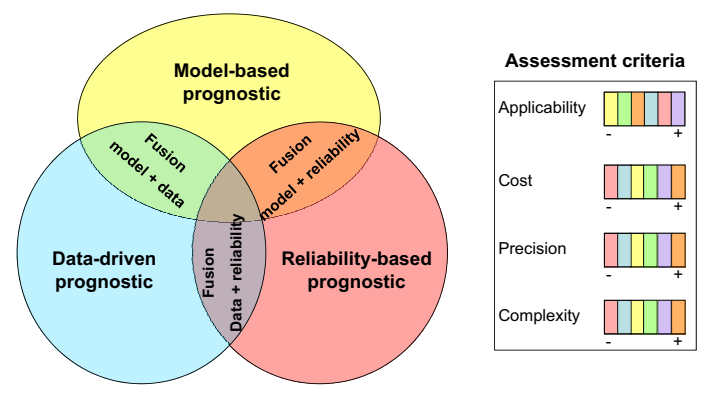

Figure 1: Classification of prognostic approaches.

based on the use of an analytical model to characterize the behavior of the system including the degradation phenomenon. The model can be a set of equations obtained by using traditional laws of physics (crack by fatigue, wearing and corrosion phenomena, etc.) $[17,18]$. The advantage of the methods used in this approach is that they provide more precise results. However, their disadvantage dwells in the fact that real systems are often nonlinear and the degradation mechanisms are generally stochastic and difficult to obtain in the form of analytical models. Consequently, the applicability of this approach may be limited by the modeling hypothesis. Reliability-based prognostic methods use mainly the data of the experience feedback gathered during a significant period of time (maintenance and operating data, failure times, etc.) in order to adjust the parameters of some reliability models (Weibull law, exponential law, etc.). These latter are then used to estimate the RUL [19]. The reliability prognostic methods are the simplest to implement when a significant amount of experience feedback data is available. The main advantage of these methods is that they do not use on-line sensors' data, neither complex mathematical models. Instead, they are rather based on the use of simple reliability functions. However, the prognostic results they offer are less precise than those provided by model-based and data-driven approaches, especially when the operating conditions are variable or in the case of new systems because of a lack of experience data. Data-driven methods attempt to transform the raw monitoring data into relevant information and behavior models of the system including the degradation instead of building mod- els based on comprehensive system physics and human expertise. They use artificial intelligence (AI) tools (neuronal networks, Bayesian networks, hidden Markov models, etc.) $[20,21]$ or statistical models to learn the degradation model $[22,23]$ to predict the future health state and the associated RUL of the system. The principle of these methods consists in two stages: a first phase during which a behavior model is learned from the monitoring data and a second phase where the learned model is used to estimate the current health condition of the system and to predict its future state. These methods can be considered as a trade-off between the model-based and the experience-based methods. This is because, on one hand, in real industrial applications getting reliable data is easier than constructing physical or analytical behavior models. On the other hand, the generated behavioral models from real monitoring data lead to more precise prognostic results than those obtained from experience feedback data.

In addition to the aforementioned prognostic approaches, three fusion ones, resulting from the intersection of the main approaches, have been reported in the recent literature. They are named "model-based/data-driven fusion", "model-based/reliability fusion" and "data-driven/reliability fusion". Model-based/data-driven methods use mathematical and physical models of a degradation and parametric models obtained from artificial intelligence and advanced statistics to build accurate models. Unfortunately, the implementation of these methods are cost and time consuming. In this category, one can cite [24] where the authors proposed a fatigue degradation model using the well-known Paris-Erdogan law coupled with a particle filtering model to estimate the failure probability, and built a cost per unit time variable to choose the best replacement time.

The model-based/reliability fusion approach offers a good accuracy, due to the use of mathematical models, and a long forecasting time horizon by the integration of the experience feedback data. In this field the works of Kacprzynski et al. $[18,25]$ deserve to be mentioned. The authors used the Paris law to determine the crack length in a helicopter's gearbox pinion, combined with reliability equations to confirm the degradation phase and to refine the fatigue propagation parameters.

Finally, data-driven/reliability fusion approach combines the ability of data-driven models to capture the non linearity and the transient phenomena associated to the real world process by using machine learning techniques. These artificial intelligence models merged with the reliability data allows to make a good forecasting for a long time horizon. In this category, one can cite the works of Heng et al. [26] where the KaplanMeier estimator is used to model complete and incomplete monitoring data histories. The probabilities given by the estimator and the data taken from the monitoring system are used in a Feed Forward Neuronal Network (FFNN) to estimate the probability of asset survival for a long future time horizon.

In this paper a data-driven prognostic method based upon the use of Mixture of Gaussians Hidden Markov Model (MoGHMM) and the wavelet packet decomposition for feature extraction is proposed. The use of this AI tool is motivated by 
the fact that it permits to handle complex emission probability density functions (pdfs) generated by a set of continuous features extracted from raw monitoring signals using the WPD that generalize the wavelet transform. The method, presented in section 3, is performed in two steps: an off-line step where the raw data are used to learn a behavioral model of the physical component's condition, and an on-line step in which the learned model is used to identify the current condition of the component and to estimate its RUL. In section 4 the method is tested on real operating data related to bearings, and simulation results are given.

\section{BACKGROUND}

A brief introduction to the fundamentals required to deploy the method are presented in this section. First the WPD for feature extraction is presented and then the MoG-HMM used to build the models is exposed.

\section{Wavelet Packet Decomposition}

Wavelet packet decomposition is now becoming a competent tool for signal analysis. Compared with the normal wavelet analysis, it has special abilities to attain higher discrimination by analyzing the higher frequency domains of a signal. The frequency domains separated by the wavelet packet can be easily selected and classified according to the characteristics of the analyzed signal. So the wavelet packet is more appropriate than wavelet in signal analysis and has much wider applications such as signal and image compression, de-noising and speech coding [27].

Wavelet packet decomposition uses a pair of low pass and high pass filters to divide a space; this corresponds to splitting the frequency content of a signal into approximately a low-frequency and a high-frequency component. Wavelet decomposition leaves the high-frequency part alone and keeps splitting the low-frequency part. In wavelet packet decomposition, we can choose to split the high-frequency part also into a low-frequency part and a high-frequency part. Thus, in general, wavelet packet decomposition divides the frequency space into various parts and allows better frequency localization of signals [28].

As shown in Fig. 2, the wavelet packet decomposition can be viewed as a tree. The root of the tree is the original data set. The next level of the tree is the result of one step of the wavelet transform. Subsequent levels in the tree are constructed by recursively applying the wavelet transform step to the low and high pass filter results from the previous wavelet transform step. Then when the decomposition process is achieved the energy in the different spectrum bands can be calculated.

\section{Mixture of Gaussians Hidden Markov Models}

A MoG-HMM is primarily an Hidden Markov Model (HMM). In [29] an HMM is defined as a statistical model used to represent stochastic processes where the states are not directly observed, see Fig. 3.

An HMM is completely defined by the following parameters:
- N: number of states in the model. The individual states are $1,2, \ldots, N$, and the state at time $t$ is defined as $s_{t}$.

- M: the number of distinct observations for each state. The observation symbols correspond to the physical output of the modeled system. The individual observation symbols are denoted as $V=v_{1}, v_{2}, \ldots, v_{M}$.

- A: the state transition probability distribution, $A=a_{i j}$, where $a_{i j}=P\left[s_{t+1}=j \mid s_{t}=i\right], 1 \leq i, j \leq N$.

- B: the observation probability distribution of a state $i, B=$ $b_{i}(k)$, where $b_{i}(k)=P\left[v_{k} \mid s_{t}=i\right], 1 \leq i \leq N, 1 \leq k \leq$ M.

- $\pi$ : the initial state distribution $\pi=\pi_{i}$, where $\pi_{i}=$ $P\left[s_{l}=i\right], 1 \leq i \leq N$.

For simplicity and clarity of presentation, a compact notation $(\lambda=\pi, A, B)$ is used for each HMM. In practice, HMMs are used to solve the following problems [29]:

- Problem 1 (detection): given a model $\lambda$ and an observation sequence $O=\left(O_{1}, O_{2}, \ldots, O_{T}\right)$, compute the probability $P(O \mid \lambda)$ of the sequence given the model. The solution of this problem is obtained by using the forward-backward algorithm [30].

- Problem 2 (decoding): given an observation sequence $O=\left(O_{1}, O_{2}, \ldots, O_{T}\right)$, find the hidden state sequence $S=$ $\left(S_{1}, S_{2}, \ldots, S_{T}\right)$ that have most likely produced the observation sequence. This problem is solved by using the Viterbi algorithm [31].

- Problem 3 (learning): find the model parameters $(\pi, A, B)$ that better fit the observation sequence $O$, i.e., that maximize the probability $P(O \mid \lambda)$. To solve this problem a solution is proposed by using the Baum-Welch algorithm [32].

Usually, HMMs consider the observations as discrete symbols and use discrete probability densities to model the transition and the observation probabilities. The problem with this approach is that in condition monitoring the observations are typically continuous signals. In order to use a continuous observation density, some restrictions are placed to insure that the parameters of the probability density function are reestimated. The most general representation of the pdf, for which a re-estimation procedure has been formulated [29], is a finite mixture of pdfs in the form:

$$
b_{i}(O)=\sum_{m=1}^{M} C_{j m} \xi\left(O, \mu_{j m}, U_{j m}\right), 1 \leq j \leq N
$$

Where $O$ is the observation vector, $C_{j m}$ is the mixture coefficient for the $m^{t h}$ mixture in state $i$ and $\xi$ is any log concave or elliptically symmetric density (e.g. Gaussian) with mean vector $\mu_{j m}$ and covariance matrix $U_{j m}$ for the $m^{t h}$ mixture component in state $j$. Usually a Gaussian density is used for $\xi$ and the corresponding model is called a MoG-HMM, that is completely defined by the parameters: the $A$ matrix, the $B$ matrix and the initial probability $\pi$. For a MoG-HMM the observation matrix $B$ is modeled by a Gaussian density with a mean $\mu$, a standard deviation $\sigma$ and a mixture matrix $M$. 


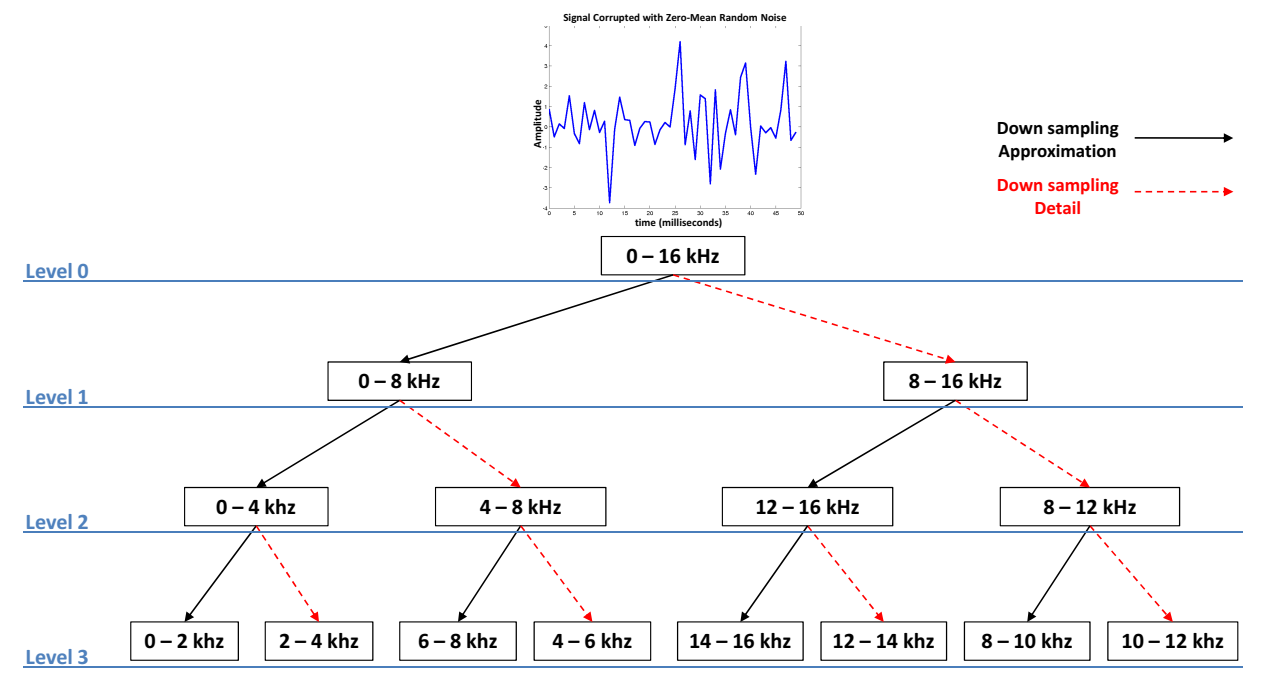

Figure 2: Wavelet packet decomposition tree.

\section{WPD/MOG-HMM BASED PROGNOSTIC METHOD}

An unified diagnostic and prognostic method to evaluate the current health state of a physical component and its remaining useful life is proposed in this section. The method is based on a nondestructive control and uses the data provided by sensors installed to monitor the component's condition. The originality of the proposed method dwells in the fact that raw signals are processed using the WPD to extract the relevant information to learn the behavior models. Also in the generated MoG-HMM the states' stay durations are not assumed to be a geometrically decaying functions [33] (which is the case in traditional HMM based prognostic methods), but are learned from the monitoring data (note that multiple observations are considered for both learning and simulation phases, instead of the traditional mono observation approach). Moreover, in the proposed method there is no limitation for the type of the generated MoG-HMM (the model can be an ergodic, a left-right or a parallel left-right). The principle of the proposed method relies on two main stages, as shown in Fig. 4: a learning phase and an exploitation phase.

In the first phase, which is executed off-line, the raw data recorded by the sensors are processed in order to extract the

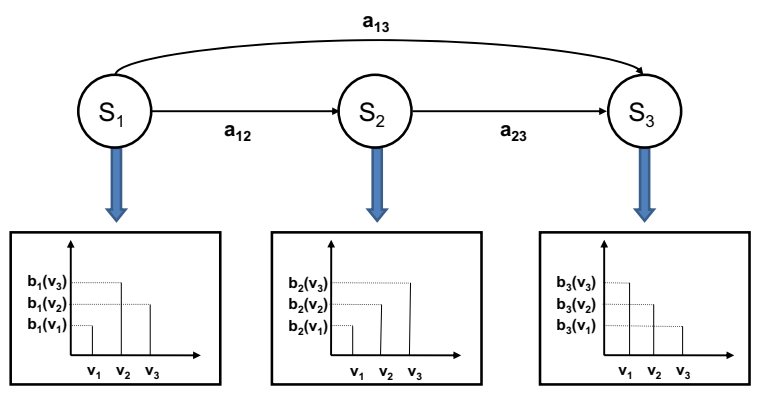

Figure 3: A three state left-to-right HMM.

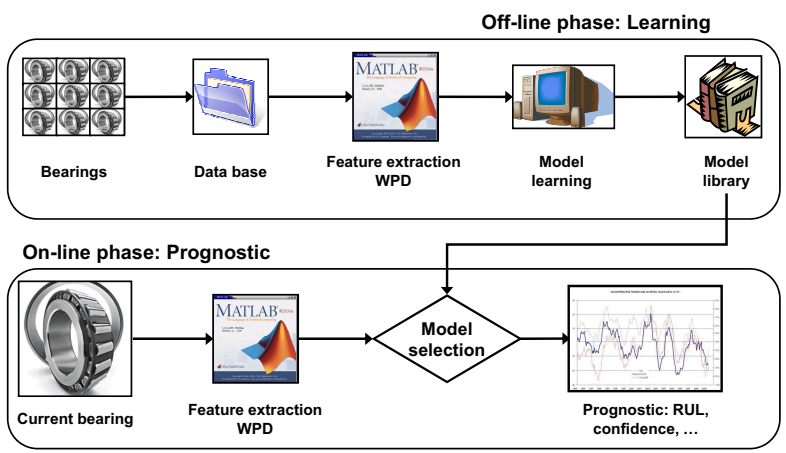

Figure 4: General diagnostics and prognostics process steps.

energy of each node at the last decomposition level [28] using the WPD. These features are then used to learn several behavior models (in the form of MoG-HMMs) corresponding to different initial states and operating conditions of the component. Indeed, each raw data history corresponding to a given component's condition is transformed to a feature matrix $F$, by using the WPD. In the matrix $F$, each column vector (of $C$ features at time $t$ ) corresponds to a snapshot on the raw signal, and each cell $f_{c t}$ represents the node $c$ of the last WPD level at time $t$.

$$
\begin{aligned}
& \text { Raw signal } \stackrel{W P D}{\longrightarrow} F=\left(f_{1 t} f_{2 t} \cdots f_{c t}\right)^{\prime} \\
& \text { with } 1 \leq t \leq T \text { and } 1 \leq c \leq C
\end{aligned}
$$

The nodal energy (features) are then used to estimate the parameters ( $\pi, A$ and $B$ ) and the temporal parameters (stay duration in each state) of the MoG-HMMs. The advantage of using several features instead of only one dwells in the fact that it can happen that a single feature may not capture all the information related to the behavior of the component.

The parameters ( $\pi, A$ and $B$ ) of each MoG-HMM are learned by using the well known Baum-Welch algorithm [30], whereas the temporal ones are estimated by using the Viterbi algorithm [31]. In addition, this latter permits to obtain the 
state sequence and to compute the time duration for which the component has been in each state of the corresponding MoGHMM (Fig. 5). Thus, by assuming that the state duration in each state follows a normal law, it is possible to estimate the mean duration (3) and the corresponding standard deviation (4) by computing the duration and the number of visits in each state. Moreover, the Viterbi algorithm permits to identify the final state which represents the physical component's failure state.

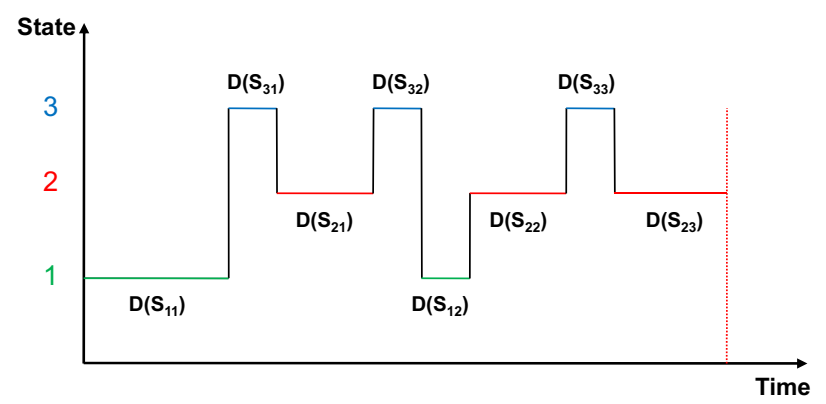

Viterbi decoding

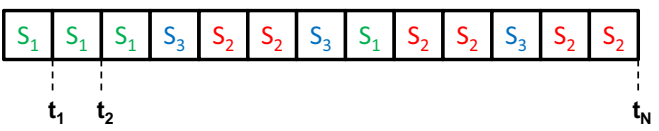

Figure 5: Example of Viterbi decoding state.

$$
\begin{gathered}
\mu\left(D\left(S_{i}\right)\right)=\frac{1}{\Omega} \sum_{w=1}^{\Omega} D\left(S_{i w}\right) \\
\sigma\left(D\left(S_{i}\right)\right)=\sqrt{\frac{1}{\Omega} \sum_{w=1}^{\Omega}\left[D\left(S_{i w}\right)-\mu\left(D\left(S_{i}\right)\right)\right]^{2}}
\end{gathered}
$$

In (3) and (4) $D(\cdot)$ stands for the visit duration, $i$ is the state index, $w$ is the visit index and $\Omega$ corresponds to the total of visits. A compact representation of each learned MoG-HMM used to perform diagnostic and prognostic is given by the following expression:

$$
\lambda=\left(\pi, A, B, \mu\left(D\left(S_{i}\right)\right), \sigma\left(D\left(S_{i}\right)\right), S_{\text {final }}\right)
$$

where $S_{\text {final }}$ is the final state (corresponding to the end of the considered condition monitoring history), $\mu\left(D\left(S_{i}\right)\right)$ is the mean state duration for the state $i$ and $\sigma\left(D\left(S_{i}\right)\right)$ is the standard deviation over the state duration for the state $i$.

The second phase, which is performed on-line, consists in exploiting the learned models to detect the component's current condition (using the Viterbi algorithm) and to compute the corresponding RUL. The processed data and the extracted nodal energy using the Wavelet toolbox from Matlab ${ }^{\circledR}$ are thus continuously fed to the learned models in order to select the one that best represents the observed data and therefore the corresponding component's condition, see Fig. 4. The selection process is based on the calculation of a likelihood $P(O \mid \lambda)$ of the model over the observations HMMs problem 1). Finally, by knowing the current condition and by using the stay durations learned in the off-line phase, the component's RUL and its associated confidence value can be estimated.

The generated MoG-HMMs are used during the on-line phase to estimate the RUL and the associated confidence value of the physical component by using a dedicated procedure whose steps are the following:

- The first step consists in detecting the appropriate MoGHMM that best fits and represents the on-line observed sequence of nodal energy. Indeed, the features are continuously fed to the set of learned models (completely defined) and a likelihood is calculated in order to select the appropriate model (Fig. 6). The selected model is then used to compute the RUL.

- The second step of this procedure concerns the identification of the current state of the component. The Viterbi algorithm is thus applied on the selected model in order to find the state sequence, which corresponds to the observed sequence of nodal energy, and to identify the current state of the component by choosing the most persistent state in the last observations (6).

$$
\begin{aligned}
& \text { state sequence }=\left(S_{1}, S_{2}, \ldots, S_{t}\right), \\
& \text { Last states }=\left(S_{t-l}, \ldots, S_{t-2}, S_{t-1}, S_{t}\right),
\end{aligned}
$$

with $l=$ past observations factor and $t=$ current time.

- The third step consists in using the current identified state, the final state (the failure state) and the probability transition matrix $A$ of the selected MoG-HMM to find the critical path, which goes from the current state to the end state. The idea is to identify all the non-zero probabilities in the transition matrix as potential transitions, and then to choose the minimal path among all the possible ones (Fig. 7) with only one visit per state. In the same way, it is possible to find the longest path by considering a maximum number of states in the path with only one visit per state. The shortest path is assimilated to the pessimistic path (rapid evolution to the failure), whereas the longest path is taken as the optimistic scenario.

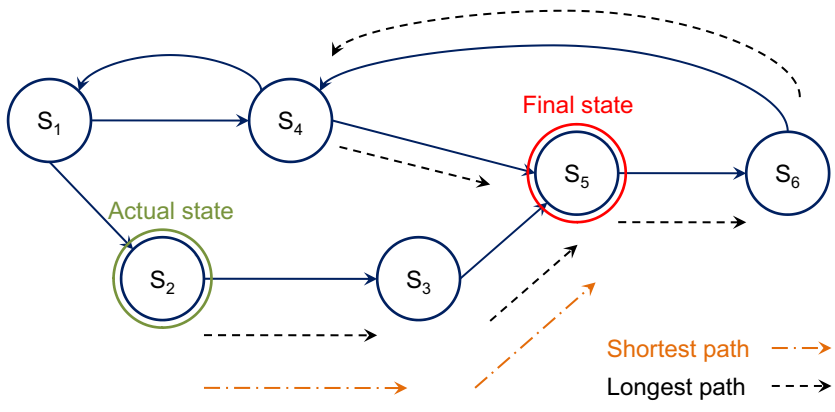

Figure 7: Path estimation. 


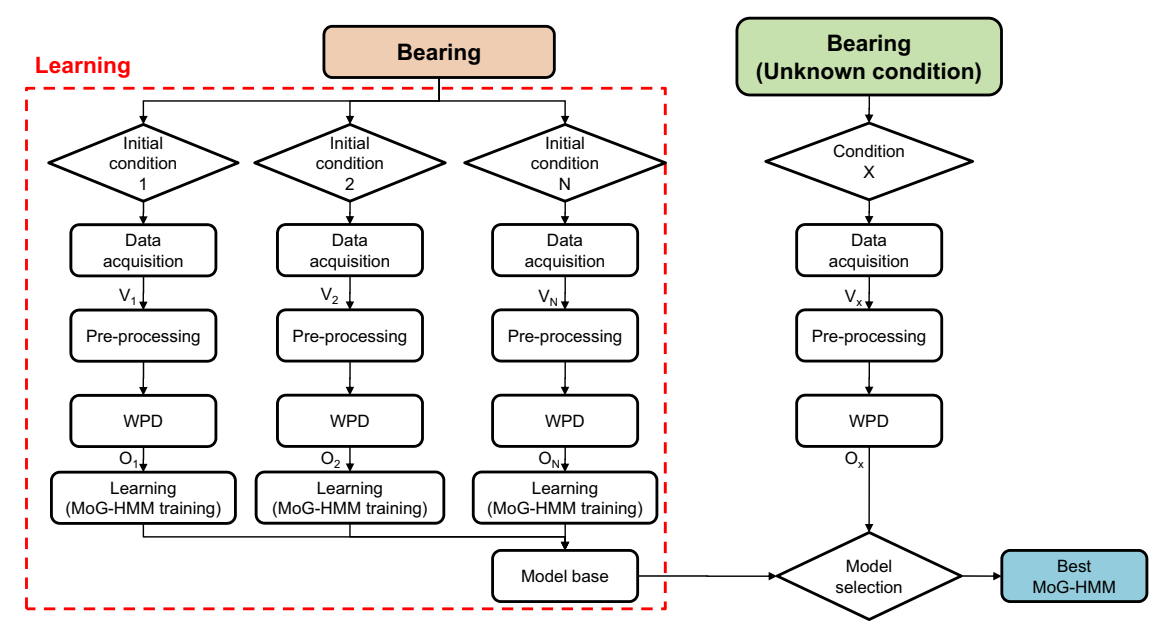

Figure 6: Competitive model selection.

- Finally, in the fourth step the paths identified previously are used to estimate the RUL. This latter is obtained using the temporal parameters of the stay duration in each state. In addition, a confidence value over the RUL is calculated based on the standard deviation values of the stay durations. Thus, three values (7) are calculated for each path: the upper RUL $(\mu+n \cdot \sigma)$, the mean RUL, and the lower RUL $(\mu-n \cdot \sigma)$.

$$
\begin{aligned}
& R U L_{\text {upper }}=\sum_{i=\text { current state }}^{N}\left[\mu\left(D\left(S_{i}\right)\right)+n \cdot \sigma\left(D\left(S_{i}\right)\right)\right], \\
& R U L_{\text {mean }}=\sum_{i=\text { current state }}^{N} \mu\left(D\left(S_{i}\right)\right), \\
& R U L_{\text {lower }}=\sum_{i=\text { current state }}^{N}\left[\mu\left(D\left(S_{i}\right)\right)-n \cdot \sigma\left(D\left(S_{i}\right)\right)\right],
\end{aligned}
$$

$\forall i \in$ state in the active path, $n=$ confidence coef.

\section{Application AND Simulation Results}

The failure prognostic method presented previously is tested on a rich condition monitoring data base taken from [34] and containing several bearings tested until the failure. The choice of bearings can be explained by the fact that these components are considered as the most common mechanical elements in industry and are present in almost all industrial processes, especially in those using rotating elements and machines. Moreover, bearings are the main components which most frequently fail in rotating machines [35] (Fig. 8). Thus, the prediction of the ETTF or RUL of these components may help improving the reliability, the availability and the safety of the rotating machines while reducing their maintenance costs, and the operational impact and environmental impact.

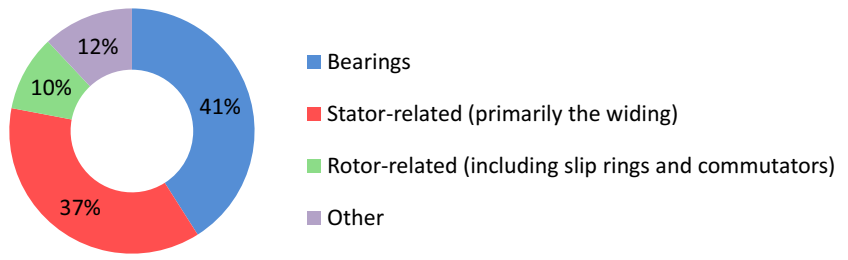

Figure 8: Failure distribution of motors of power greater than $200 \mathrm{hp}$.

The test data extracted from [34] correspond to several tests under constant conditions. Four bearings were installed on one shaft. The angular velocity was kept constant at 2000 rpm and a $6000 \mathrm{lb}$ radial load was applied onto the shaft and bearings (Fig. 9). On each bearing two Accelerometer were installed for a total of 8 accelerometers (one vertical $\mathrm{Y}$ and one horizontal $\mathrm{X}$ ) to register the accelerations generated by the vibrations, the sampling rate was fixed at $20 \mathrm{kHz}$. For simulation purposes (learning and on-line failure prognostic) twelve condition monitoring data histories are used (eleven for learning and one for test), each bearing was considered failed at the end of its associated history. For both learning

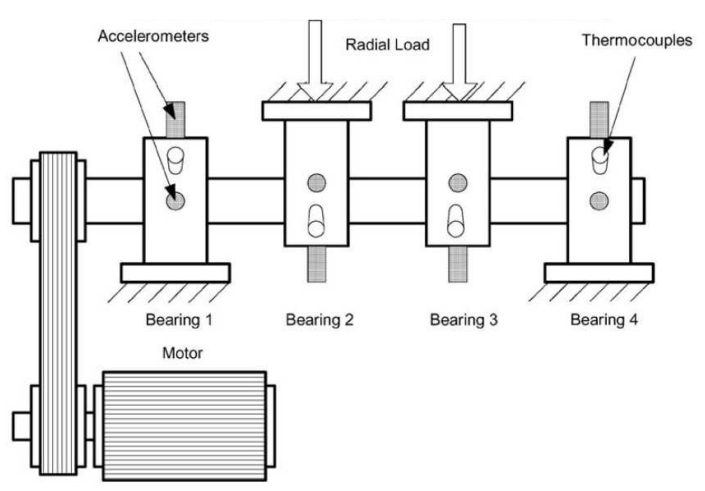

Figure 9: The bearing test bed [34]. 
and prognostic phases, the nodal energy in third level of the WPD (using the "Daubechies" wavelet family) at each instant $t$ have been extracted from the raw signals (vibration signals). The level chose using (8) that defines the maximum decomposition level where at lest 3 harmonics of the characteristic defect frequency are caught. In this expression $J_{f}$ is the decomposition level, $F_{s}$ the sampling frequency and $F_{d}$ the defect frequency. For this case the fault frequency is the band pass frequency of the outer race (BPFO) this suggest a decomposition level of 3.8. Being conservative the decomposition level is fixed to three. This choice permits to obtain wide frequency bands of $2.5 \mathrm{kHz}$. The principle of the procedure for feature extraction is shown in Fig. 10.

$$
J_{f} \leqslant \log _{2} \frac{F_{s}}{3 F_{d}}-1
$$

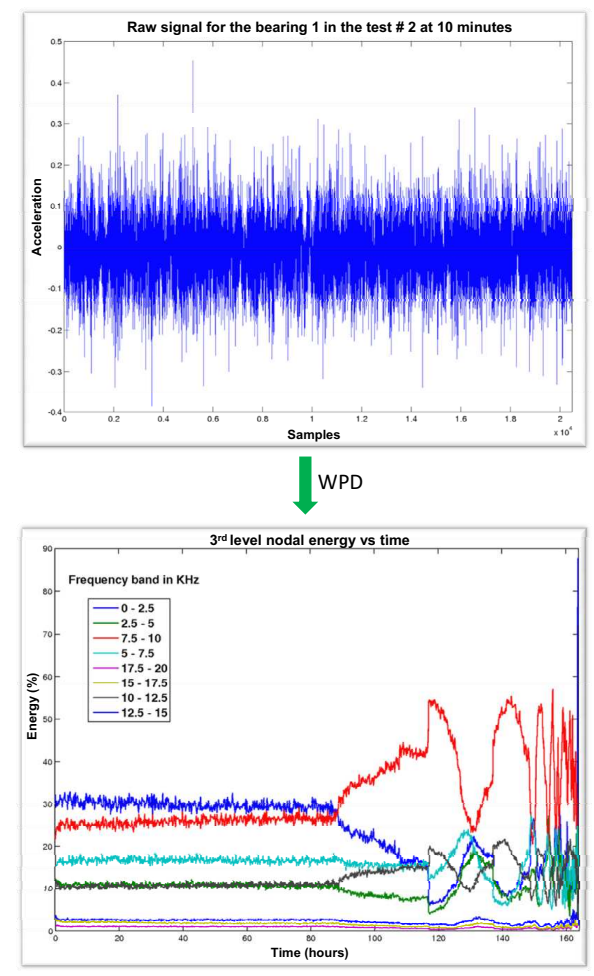

Figure 10: Feature extraction principle.

During the learning phase, three states are defined for each MoG-HMM. The parameters of each MoG-HMM are first randomly initialized and then the continuous extracted features are fed to the learning algorithms in order to re-estimate the initialized parameters $(\pi, A$ and $B)$. The number of mixtures in each MoG-HMM is set to two, which allows a tradeoff between precision and computation time. Eleven MoGHMMs are thus obtained by using the Baum-Welch algorithm. The re-estimated numerical values of the parameters $\pi, A$ and $M$ of a MoG-HMM related to bearing one in the test $\mathrm{N}^{\circ} 1$ are : $\pi=\left(\begin{array}{l}0 \\ 1 \\ 0\end{array}\right), A=\left(\begin{array}{ccc}0.98 & 0.01 & 0.01 \\ 0.01 & 0.99 & 0 \\ 0.02 & 0 & 0.98\end{array}\right), M=\left(\begin{array}{cc}0.18 & 0.82 \\ 0.86 & 0.14 \\ 0.58 & 0.42\end{array}\right)$

The mean state duration, the standard deviation and the final state for this history are presented below.

$$
\begin{gathered}
{\left[\begin{array}{l}
\mathrm{S}_{1} \\
\mathrm{~S}_{2} \\
\mathrm{~S}_{3}
\end{array}\right]=\left[\begin{array}{ll}
\mu\left(\mathrm{S}_{1}\right), & \sigma\left(\mathrm{S}_{1}\right) \\
\mu\left(\mathrm{S}_{2}\right), & \sigma\left(\mathrm{S}_{2}\right) \\
\mu\left(\mathrm{S}_{3}\right), & \sigma\left(\mathrm{S}_{3}\right)
\end{array}\right]=\left[\begin{array}{cc}
2773, & 1748 \\
1300, & 260 \\
5320, & 4280
\end{array}\right] \text { min }} \\
S_{\text {final }}=S_{2}
\end{gathered}
$$

In order to simulate an on-line failure the "test" data history is used. The selection process shown in Fig. 6 is then applied on the data history corresponding to the bearing 2 in the test $\mathrm{N}^{\circ} 2$ in order to identify the "best MoG-HMM" and to estimate the RUL. A simulation result of the predicted RUL and his error is shown in Fig. 11, where the data history used to perform the test correspond to a faulty bearing that ends at $9840 \mathrm{~min}$.

From Fig. 11-(a) and Fig. 11-(b) one can see that the precision of the estimated RUL increases as the prediction time is approaching the real failure time. Similarly, after 7440 min the mean estimation error drops below $16.5 \%$ whereas when considering the upper RUL limit the error drops to $7 \%$ and continues to decrease as the real failure time approaches. After $9610 \mathrm{~min}$ the mean error stabilizes arround the value of $1.08 \%$. The $68 \%$ confidence interval keeps the RUL estimation limits near acceptable values, a wide confidence intervals (95\% and $99.5 \%$ ) will give more sparse limits and will increase the prediction error. These results are better that those obtained in our previous work [36] where the temporal features used where: RMS, mean, skewness and kurtosis. The RUL and the associated prediction error of the same test history using temporal features are shown in Fig. 11-(c) and Fig. 11-(d). One can appreciate the strong inertia on the behavior of the prognostic results whereas the model that uses WPD for feature extraction evolves progressively converging to the real RUL.

\section{Conclusions}

An estimation of the current health condition of physical components, particularly bearings, and an estimation of their remaining useful life before their complete failure has been proposed in this paper. The method is based on the transformation of the data provided by the sensors installed to monitor the component into relevant models. These latter are represented by MoG-HMMs, which take as input continuous observations and permit to model the state of the component at each time. A WPD has been used to extract appropriate features from the monitoring signals. This type of decomposition allowed getting deeper into the signal features by adjusting both the time and the frequency scales. These features are then used to model the degradation behaviour of the compo- 


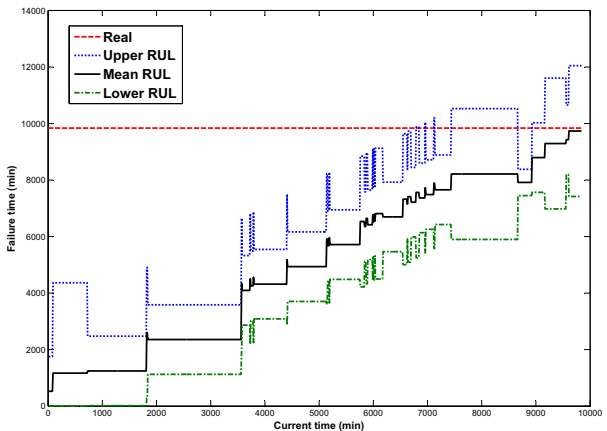

(a)

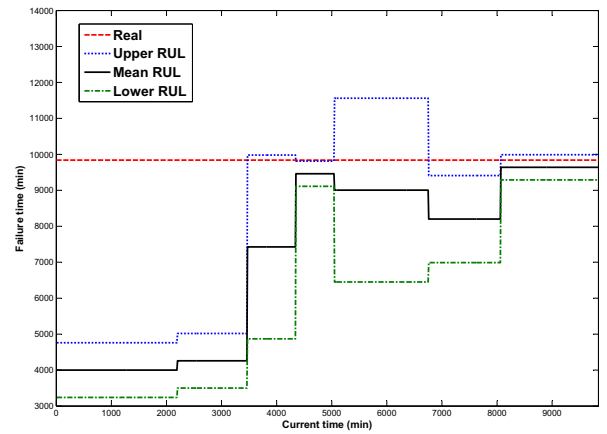

(c)

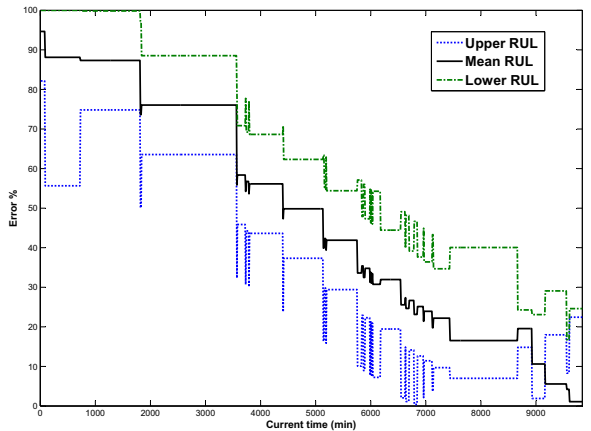

(b)

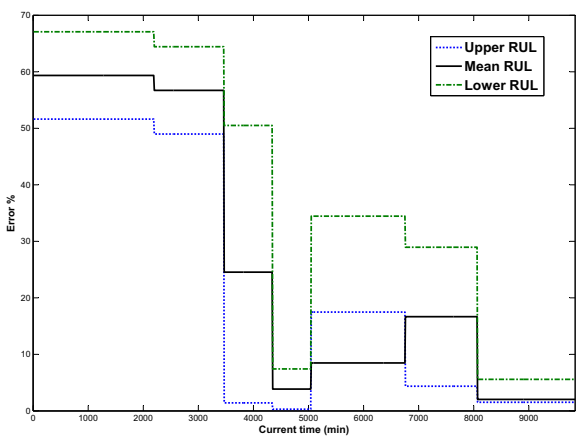

(d)

Figure 11: Simulation results: (a) RUL estimation for the bearing 2 in test $\mathrm{N}^{\circ} 2$ using WPD; (b) prediction error for the bearing 2 in test $\mathrm{N}^{\circ} 2$ using WPD; (c) RUL estimation for the bearing 2 in test $\mathrm{N}^{\circ} 2$ using temporal features; and, (d) prediction error for the bearing 2 in test $\mathrm{N}^{\circ} 2$ using temporal features.

nent by learning the parameters of the corresponding MoGHMMs models. The derived models are finally exploited to asses the current condition and to estimate the RUL and the confidence value. The time-frequency features allowed getting more precise result than using only time features as shown in the comparison done in this paper.

\section{REFERENCES}

[1] A. K. Jardine, D. Lin, and D. Banjevic, "A review on machinery diagnostics and prognostics implementing condition-based maintenance," Mechanical Systems and Signal Processing, vol. 20, no. 7, pp. 1483 - 1510, 2006.

[2] G. Vachtsevanos, F. L. Lewis, M. Roemer, A. Hess, and B. Wu, Intelligent fault diagnosis and prognosis for engineering systems. Wiley, 2006.

[3] N. Tandon and A. Choudhury, "A review of vibration and acoustic measurement methods for the detection of defects in rolling element bearings," Tribology International, vol. 32, no. 8, pp. $469-480,1999$.

[4] T. Kurfess, S. Billington, and S. Liang, "Advanced diagnostic and prognostic techniques for rolling element bearings," Springer Series in Advanced Manufacturing, pp. 137-165, 2006.

[5] G. Strang and T. Nguyen, Wavelets and Filter Banks, W.-C. Press, Ed. Wellesley College, 1996.
[6] AFNOR, "Condition monitoring and diagnostics of machines - prognostics - part 1: General guidelines. NF ISO 13381-1,” 2005.

[7] G. Provan, "Prognosis and condition-based monitoring: an open systems architecture," in IFAC Symposium on Fault Detection, Supervision and Safety of Technical Processes, no. 5, 2003, pp. 57-62.

[8] W. Q. Wang, M. F. Golnaraghi, and F. Ismail, "Prognosis of machine health condition using neuro-fuzzy systems," Mechanical Systems and Signal Processing, vol. 18, no. 4, pp. 813 - 831, 2004.

[9] A. Muller, M.-C. Suhner, and B. Iung, "Formalisation of a new prognosis model for supporting proactive maintenance implementation on industrial system," Reliability Engineering \& System Safety, vol. 93, no. 2, pp. 234 253, 2008.

[10] A. Heng, S. Zhang, A. C. Tan, and J. Mathew, "Rotating machinery prognostics: State of the art, challenges and opportunities," Mechanical Systems and Signal Processing, vol. 23, no. 3, pp. 724 - 739, 2009.

[11] D. A. Tobon-Mejia, K. Medjaher, and N. Zerhouni, "The iso 13381-1 standard's failure prognostics process through an example," in IEEE - Prognostics \& System Health Management Conference, University of Macau, Macau, China, 12-14 January 2010 2010, in Press.

[12] C. Farrar, F. Hemez, G. Park, A. Robertson, H. Sohn, 
and T. Williams, "A coupled approach to developing damage prognosis solutions," in 5th International Conference on Damage Assessment of Structures, Southampton, England, July 1-3 2003.

[13] L. D and M. V, "Recursive filters for a partially observable system subject to random failure," Adv. in Applied Probability, pp. 207 - 227, 2003.

[14] R. Isermann, "Model-based fault-detection and diagnosis - status and applications," Annual Reviews in Control, vol. 29, no. 1, pp. 71-85, 2005.

[15] V. Venkatasubramanian, "Prognostic and diagnostic monitoring of complex systems for product lifecycle management: Challenges and opportunities," Computers \& Chemical Engineering, vol. 29, no. 6, pp. 1253 1263, 2005.

[16] M. Lebold and M. Thurston, "Open standards for condition-based maintenance and prognostic systems," in Maintenance and Reliability Conference (MARCON), 2001.

[17] J. Luo, K. R. Pattipati, L. Qiao, and S. Chigusa, "Modelbased prognostic techniques applied to a suspension system," Transactions on Systems, Man, and Cybernetics, vol. 38, pp. 1156-1168, 2003.

[18] G. Kacprzynski, A. Sarlashkar, M. Roemer, A. Hess, and B. Hardman, "Predicting remaining life by fusing the physics of failure modeling with diagnostics," Journal of the Minerals, Metals and Materials Society, vol. 56, pp. 29-35, 2004.

[19] K. Goode, J. Moore, and B. Roylance, "Plant machinery working life prediction method utilizing reliability and condition-monitoring data," Proceedings of Institution of Mechanical Engineers, vol. 214, pp. 109 - 122, 2000.

[20] R. B. Chinnam and P. Baruah, "A neuro-fuzzy approach for estimating mean residual life in condition-based maintenance systems," International Journal of Materials and Product Technology, vol. 20, pp. 166 - 179, 2004.

[21] D. C. Swanson, J. M. Spencer, and S. H. Arzoumanian, "Prognostic modelling of crack growth in a tensioned steel band," Mechanical systems and signal processing, vol. 14, pp. 789-803, 1999.

[22] T. Wang, J. Yu, D. Siegel, and J. Lee, "A similaritybased prognostics approach for remaining useful life estimation of engineered systems," in International conference on prognostics and health management, 2008.

[23] Y. Li and P. Nilkitsaranont, "Gas turbine performance prognostic for condition-based maintenance," Applied Energy, vol. 86, no. 10, pp. 2152 - 2161, 2009.

[24] F. Cadini, E. Zio, and D. Avram, "Monte carlo-based filtering for fatigue crack growth estimation," Probabilistic Engineering Mechanics, vol. 24, no. 3, pp. 367 - 373, 2009.

[25] G. J. Kacprzynski, M. J. Roemer, G. Modgil, A. Pal- ladino, and K. Maynard, "Enhancement of physics-offailure prognostic models with system level features," in 2002 IEEE Aerospace Conference Proceedings, vol. 6, 2002, pp. 2919-2925.

[26] A. Heng, A. C. Tan, J. Mathew, N. Montgomery, D. Banjevic, and A. K. Jardine, "Intelligent conditionbased prediction of machinery reliability," Mechanical Systems and Signal Processing, vol. 23, no. 5, pp. 1600 - 1614, 2009.

[27] A. N. Akansu, W. A. Serdijn, and I. W. Selesnick, "Emerging applications of wavelets: A review," Physical Communication, vol. 3, no. 1, pp. 1-18, 2010.

[28] J. Zarei and J. Poshtan, "Bearing fault detection using wavelet packet transform of induction motor stator current," Tribology International, vol. 40, no. 5, pp. 763 769, 2007.

[29] L. R. Rabiner, "A tutorial on hidden markov models and selected applications in speech recognition," in Proceedings of the IEEE, vol. 77 (2), 1989, pp. 257-286.

[30] L. Baum and J. Egon, “An inequality with applications to statistical estimation for probabilistic functions of a markov process and to a model for ecology," Bulletin of the American Mathematical Society, vol. 73, pp. 360 363, 1967.

[31] A. Viterbi, "Error bounds for convolutional codes and an asymptotically optimal decoding algorithm," IEEE Transaction on Information Theory, vol. 13, pp. $260-$ 269, 1967.

[32] A. Dempster, N. Laird, and D. Rubin, "Maximum likelihood from incomplete data via the EM algorithm," Journal of the Royal Statistical Society, vol. 39, pp. 1 - 38, 1977.

[33] M. Dong and D. He, "A segmental hidden semimarkov model (hsmm)-based diagnostics and prognostics framework and methodology," Mechanical Systems and Signal Processing, vol. 21, pp. 2248-2266, 2007.

[34] NSF I/UCRC Center for Intelligent Maintenance Systems, "Prognostic data repository: Bearing data set," in http://ti.arc.nasa.gov/tech/dash/pcoe/prognostic-datarepository/, online in 2010.

[35] P. O'Donnell, "Report of large motor reliability survey of industrial and commercial installations, part I, II \& III," IEEE Transactions on Industry Applications, vol. 21, pp. 853-872, 1985.

[36] D. A. Tobon-Mejia, K. Medjaher, N. Zerhouni, and G. Tripot, "A mixture of gaussians hidden markov model for failure diagnostic and prognostic," in IEEE Conference on Automation Science and Engineering, CASE'10, 2010. 


\section{BIOGRAPHY}

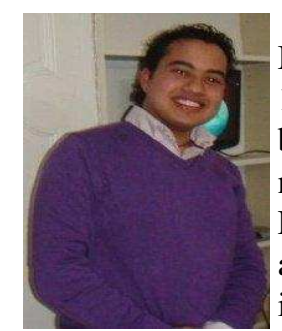

D. A. Tobon-Mejia was born in Medellin, Colombia, on February 16, 1985. He received the B.Sc. and S.M., both in 2008, from the National Engineering School in Metz (France) and the EAFIT University (Colombia). He made a specialization in his last school year in research and development in "Design, industrialisation and innovation" supported by the Paul Verlaine University, the ENSAM and the ENIM, all in Metz (France). In his last year of Master he developed a decision support application based in fuzzy logic and the PERT method to assess the failure mode hazard in a Francis turbine that works in a hydroelectric power station in Colombia, to ensure the systems availability and security. Actually he performs his doctoral studies at Franche-Comt University, in Besanon (France) sponsored by Alstom Transport where he his a research engineer. He is engaged in research on rotating machinery failure prognostic at the FEMTO-ST institute and Alstom transport. Diego A. Tobon-Mejia, was honored in 2002 with the "Excellence scholarship" from the "EEPP de Medellin" (Medelln Public Enterprises) to perform his studies in Colombia. In 2006 he was honored by the The French Ministry of Foreign affairs with the "Eiffel Excellence scholarship" to continue his studies in France.

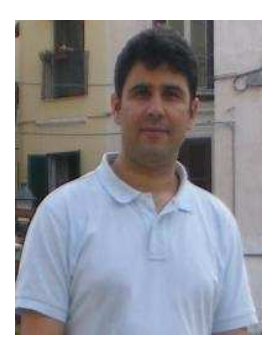

K. Medjaher is an associate Professor at the French high school of mechanics and micro-techniques in Besanon since September 2006, where he teaches control and fault diagnostics and prognostics. After an engineering degree in electronic, he has got his MS in control and industrial computing in 2002 at the "Ecole Centrale de Lille" and his PhD in 2005 in the same field from the University of Lille 1. Before joining his current position, Dr. Medjaher has mainly worked in the field of Fault Detection and Isolation (FDI). He has particularly used the bond graph formalism to derive mathematical models of physical systems and to generate Analytical Redundancy Relations (ARRs) and residuals, which are then used to detect and to isolate possible faults on the system. Since September 2006, Dr. Medjaher leads research works in the field of failure prognostics and uses artificial intelligent tools, particularly Dynamic Bayesian Networks (DBNs).

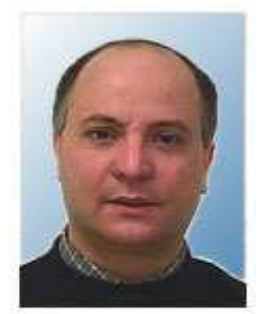

N. Zerhouni received his engineer degree from National Engineers and Technicians School of Algiers (ENITA) in 1985. After a short period in industry as engineer, he received his Ph.D. Degree in Automatic Control from the Grenoble National Polytechnic Institute in 1991. In September 1991, he joined the National Engineering School of Belfort (ENIB) as Associate
Professor. At this time, his main research activity was concerned with modelling, analysis and control of manufacturing systems. Since September 1999, Noureddine Zerhouni is Professor at the national high school of mechanics and microtechniques of Besanon. He founded and is responsible of the research group "Design and maintenance of mechatronic systems" (COSMI) of AS2M Department within FEMTO-ST Institute. His main research activities are concerned with intelligent maintenance systems and e-maintenance. Professor Noureddine Zerhouni has been and is involved in various European and National projects on intelligent maintenance systems like FP5 European Integrated Project of ITEA program (Information Technology for European Advancement) PROTEUS, NEMOSYS (Naval E-Maintenance Oriented SYStem) with DCNS, and AMIMAC-FAME (Reliability Improvement of Embedded Machines) with ALSTOM and CEGELEC.

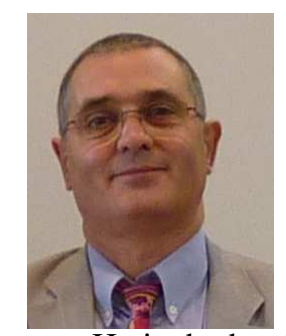

G. Tripot received his engineer degree from the "Ecole Catholique des Arts et Mtiers" of Lyon in 1980. After various position in the field of mechanical engineering, reliability and maintenability studies, materials and technologies validation, he is actually in charge of several R\&D projects within ALSTOM Transport. He is the head of the project "FAME" (Reliability Improvement of Embedded Machines) where he develops an embedded platform for predictive machine degradation assessment, remote monitoring and failure prognostics of traction motors. He serves as senior expert for various other projects, providing expertise in development and validation of new technologies. 